\title{
Efficacy of procaine combined with ketamine and propofol in pediatric epidural anesthesia
}

\author{
HAI HUANG $^{1 *}$, YUJIE LIU $^{1 *}$, ZIJUN GAO ${ }^{2}$ and XUCAI WU ${ }^{2}$ \\ ${ }^{1}$ Department of Anesthesiology, Second Affiliated Hospital of Tianjin University of Traditional Chinese Medicine, \\ Tianjin 300150; ${ }^{2}$ Department of Anesthesiology, Hong Hui Hospital, Xi'an Jiaotong University College of Medicine, \\ Xi'an, Shaanxi 710054, P.R. China
}

Received October 23, 2019; Accepted July 21, 2020

DOI: $10.3892 /$ etm.2020.9151

\begin{abstract}
In the present study, the effect of procaine combined with ketamine and propofol in pediatric epidural anesthesia was analyzed. A total of 74 children scheduled to undergo surgery under epidural anesthesia were included in the study, and were divided into two groups using a random number table. Accordingly, 37 patients received epidural anesthesia using ketamine and propofol (control group), whereas the remaining patients received procaine combined with ketamine and propofol (observation group). The vital signs, sedative effects, anesthetic effects, pain intensity, anesthetic dose and incidence of adverse reactions were compared between the groups. It was revealed that the heart rate and mean arterial pressure at $\mathrm{T} 4$ (following epidural administration) in the observation group was significantly lower compared with those of the control group $(\mathrm{P}<0.05)$. However, no statistical difference was observed in the proportion of patients with sedation at Ramsay levels 1-6 between the observation and the control group $(\mathrm{P}>0.05)$. Compared with the control group, the observation group reported a shorter latent period for the onset of the anesthetic effect and the disappearance of pain, and a longer period for the recovery of pain sensation $(\mathrm{P}<0.05)$. The observation group exhibited prominently lower visual analog scale scores at $6,12,18$ and 24 h post-surgery compared with the control group $(\mathrm{P}<0.05)$. Additionally, the observation group received a lower dose of ketamine and propofol compared with the control group $(\mathrm{P}<0.05)$. An incidence of adverse reactions of $8.11 \%$ was noted in the observation group during the surgery and anesthesia recovery period, which was lower
\end{abstract}

Correspondence to: Dr Xucai Wu, Department of Anesthesiology, Hong Hui Hospital, Xi'an Jiaotong University College of Medicine, 555 Youyi East Road, Nanshaomen, Beilin, Xi'an, Shaanxi 710054, P.R. China

E-mail: jn9026s2@163.com

*Contributed equally

Key words: children, epidural anesthesia, procaine, ketamine, propofol compared with that of the control group $(29.73 \%)(\mathrm{P}<0.05)$. The results of the present study indicated that the combination of procaine with ketamine and propofol in pediatric epidural anesthesia may be more commonly employed, considering its advantages in accelerating the anesthesia process, improving the anesthetic effects and guaranteeing anesthesia safety. However, only a few indicators of the anesthesia efficacy and a small group of patients were included in the present study, and a long-term comprehensive analysis using a larger sample size is required to address this issue.

\section{Introduction}

Anesthesia is a key process in the surgical treatment of children considering their limited cooperation, and it is also a factor that determines the success of the surgery without endangering the children. For pediatric surgery, several options for anesthesia are available. Among them, epidural anesthesia is more extensively used based on its satisfactory muscle relaxation and thorough pain-relieving effects (1).

A variety of anesthetics have been used to achieve pediatric epidural anesthesia, including propofol and ketamine, which have been indicated to be preferable in the clinical setting, among which propofol has been reported to exhibit a good sedative effect and a rapid working mechanism, and patients who receive propofol have been indicated to exhibit a quick recovery from anesthesia post surgery without significant adverse reactions (2). Despite its satisfactory performance in anesthesia, pain relief and sedation, ketamine administration has been indicated to result in an increased release of catechol and consequently in increased cardiovascular excitation during the recovery period; therefore, children may experience various side effects, such as nausea, vomiting, restlessness and nightmares (3). Currently, an auxiliary application of general anesthetics or sedative drugs is required in epidural anesthesia. Procaine, a local anesthetic commonly used in clinical practice, can stabilize the cell membrane and reduce its permeability to ions, so that when the nerve impulse reaches, sodium and potassium ions cannot move in and out of the cell membrane to generate depolarization and action potentials, thus producing a local anesthetic effect $(4,5)$. Procaine has poor penetration of mucous membrane and is not suitable for surface anesthesia, but it is less toxic than morphine and has a definite effect, 
which is also suitable for infiltration anesthesia, block anesthesia and epidural anesthesia (6). Procaine can be hydrolyzed by esterase in plasma and converted into para-aminobenzoic acid and diethylaminoethanol, where the former can resist the antibacterial effect of sulfonamides and should therefore be avoided to use with sulfonamides (7).

The present study specifically analyzed the effect of procaine in combination with ketamine and propofol in pediatric epidural anesthesia by comparing patients receiving ketamine and propofol with patients receiving procaine combined with ketamine and propofol, aiming to identify more effective and safe options for clinical pediatric epidural anesthesia.

\section{Patients and methods}

Patients. A total of 74 children with inguinal hernias, who were subjected to herniorrhaphy under epidural anesthesia in the Hong Hui Hospital (Xi'an, China) between June 2018 and September 2019 were included in the present study and divided into two groups using a random number table. The control group included 20 males and 17 females with a youngest age of 6 months and an oldest age of 18 years, who received ketamine and propofol for epidural anesthesia. The observation group included 22 males and 15 females with a youngest age of 4 months and an oldest age of 18 years, who received procaine combined with ketamine and propofol for epidural anesthesia. The inclusion criteria were as follows: Children with i) American Society of Anesthesiologists (ASA) classification grade I (8) who underwent no other treatment prior to participation in the present study; ii) who required surgery under epidural anesthesia; iii) whose general condition was verified to be satisfactory and stable; and iv) whose parents provided written informed consent. The study was approved by the Ethics Committee of Hong Hui Hospital, Xi'an Jiaotong University College of Medicine (Xi'an, China). Children with a history of non-surgical treatment, anesthesia by means other than epidural anesthesia, ASA grade II or above, and anesthesia contraindications and concurrent cardiac, hepatic, pulmonary and renal dysfunction were excluded from the study.

Anesthesia. According to the clinical experience of the hospital and a previous study, epidural anesthesia was determined as the preferred anesthesia method in the current study (9). Before surgery, all patients received an intramuscular injection of atropine $(0.02 \mathrm{mg} / \mathrm{kg}$, approval no. GYZ Zi H32020166; Jiangsu Lianshui Pharmaceutical Co., Ltd.), diazepam (0.2 mg/kg, approval no. GYZ Zi H14022662, manufactured by Shanxi Zhendong Pharmaceutical Co., Ltd.) and ketamine (4-5 mg/kg, approval no. GYZ Zi H20054748; Xi'an Hanfeng Pharmaceutical Co., Ltd.) for basic anesthesia, and a venous access was established when they entered a sleeping or quiescent state. Routine disinfection was performed in a right lateral decubitus position. Following the accurate placement of sterile towels, puncturing was implemented between the L2 and L3 spinal segments until a sense of penetration was perceived and positive return air test results were obtained. Subsequently, local anesthesia using $1 \%$ lidocaine $(5 \mathrm{mg} / \mathrm{kg}$, approval no. GYZ Zi H31021071; Shanghai Zhaohui Pharmaceutical Co., Ltd.), $0.375 \%$ bupivacaine $(5 \mathrm{mg} / \mathrm{kg}$, approval no. GYZ Zi H20056442; Shanghai Zhaohui Pharmaceutical Co., Ltd.) and
1/200,000 epinephrine $(0.25-1 \mathrm{mg} / \mathrm{kg}$, approval no. GYZ Zi H14020817; Shanxi Zhendong Pharmaceutical Co., Ltd.) was concomitantly administered before unplugging the epidural needle. The ratio of lidocaine/bupivacaine was 1:1 and the anesthesia plane did not exceed T8.

The control group was anesthetized using ketamine and propofol (approval no. H20130535; AstraZeneca UK Ltd.). Prior to skin incision, ketamine (1-2 mg/kg) was slowly injected intravenously, and an intermittent intravenous injection of propofol $(1 \mathrm{mg} / \mathrm{kg})$ combined with ketamine (1-2 $\mathrm{mg} / \mathrm{kg})$ using the same approach at half the initial dose was maintained during surgery, starting from 10-15 min after the initiation of surgery until its completion. Ephedrine (total dose 10-12 mg/kg, approval no. GYZ Zi H50020872, manufactured by Chongqing Dikang Changjiang Pharmaceutical Co., Ltd.) was administered in case of a sharp decrease in blood pressure (BP) and atropine (total dose $0.5-1.0 \mathrm{mg} / \mathrm{kg}$ ) was intravenously injected to treat a decrease in heart rate (HR). An oxygen mask was constantly used during the surgery, which was supported by manually assisted respiration when required.

The observation group was anesthetized using procaine (specification, 0.5 g; approval no. GYZ Zi H20020082; Jincheng Haisi Pharmaceutical Co., Ltd.) combined with ketamine and propofol. Prior to skin incision, ketamine (1-2 $\mathrm{mg} / \mathrm{kg}$ ) was slowly injected intravenously, and an intermittent intravenous injection of propofol $(1 \mathrm{mg} / \mathrm{kg})$ was maintained during surgery. In addition, $2.0 \%$ procaine (total dose $5 \mathrm{ml} / \mathrm{kg}$ ) along with ketamine (1-2 mg/kg) using the same approach at half the initial dose was intravenously injected, starting from 10-15 min after the initiation of surgery until its completion. Ephedrine (total dose $10-12 \mathrm{mg} / \mathrm{kg}$ ) was administered in case of a sharp decrease in BP and atropine (total dose $0.5-1.0 \mathrm{mg} / \mathrm{kg}$ ) was intravenously injected to treat a decrease in HR. An oxygen mask was constantly used during surgery. Fig. 1 depicts a flow chart presenting the research procedure of the current study.

\section{Observation indices}

Vital signs. HR and mean arterial pressure (MAP) were measured in the observation and control groups before anesthesia (T1), at 5 min after anesthesia (T2), before epidural administration (T3) and at 5 min after epidural administration (T4).

Sedative effects. The sedative effects were assessed according to Ramsay's criteria for myalgic encephalomyelitis (10), with six levels as follows: Level 1, the patient is anxious and agitated or restless or both; level 2, the patient is cooperative, oriented and tranquil; level 3, the patient responds to commands only; level 4, the patient is asleep and exhibits a rapid response to a light glabellar tap; level 5, the patient is asleep and exhibits a slow response to a light glabellar tap; and level 6 , the patient is asleep and exhibits no response to a light glabellar tap. Level 3 or above was considered to indicate satisfactory sedative effects.

Anesthetic effects. Both groups were compared in terms of the latent period for the anesthetic effect, disappearance of pain and recovery of pain sensation, which were respectively defined as follows: Time from epidural injection to perianal 


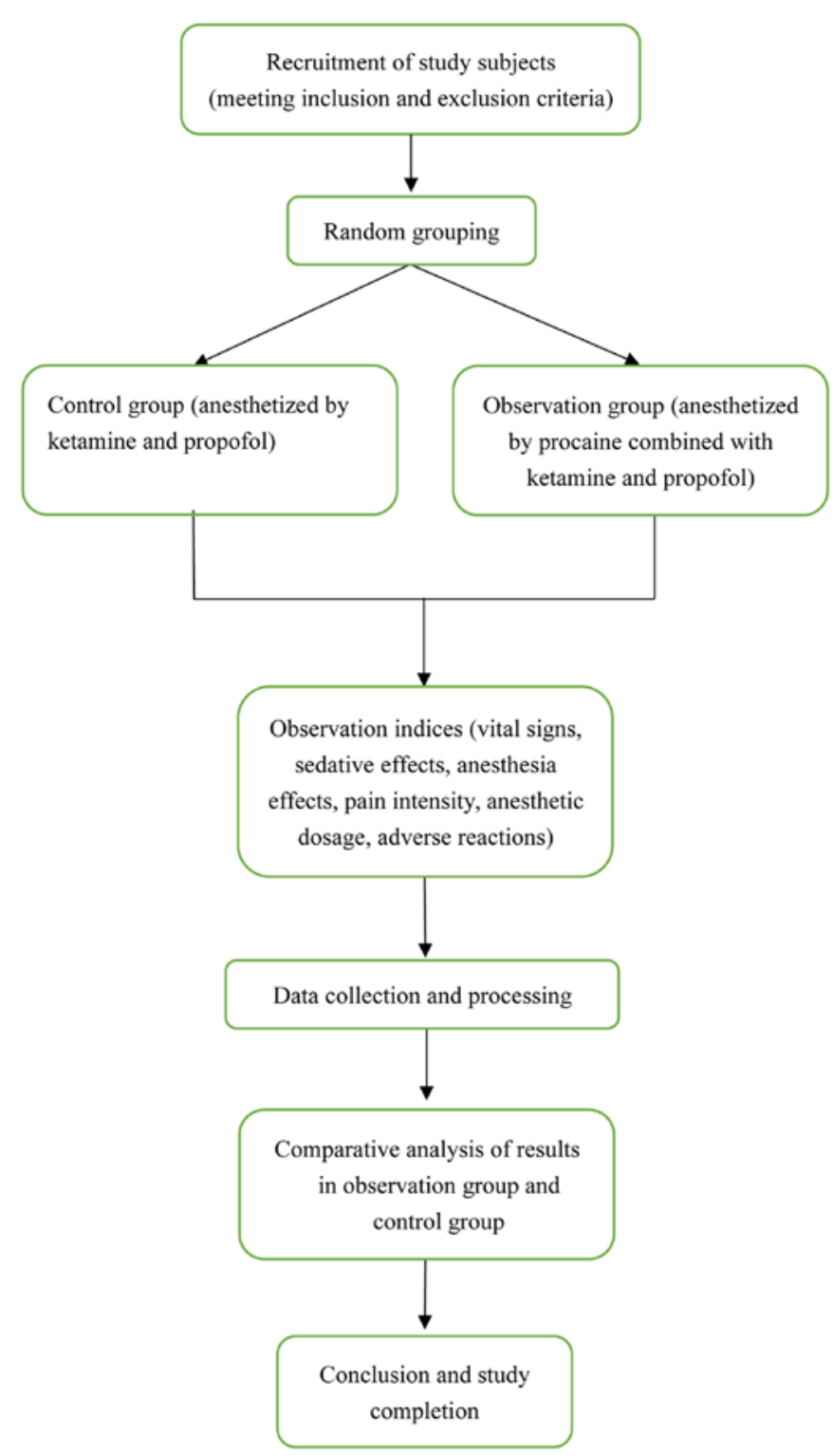

Figure 1. Flow chart of the research procedure.

superficial reflex or declined abdominal reflex, time from epidural injection to no response when the surgical site was punctured and time from epidural injection to the time when the patients regained consciousness and complained of pain or when an evident writhing in the limbs was observed despite the patients being unconscious.

Pain intensity. An 11-point visual analog scale (VAS; 0-10, no pain to worst possible pain) (11) was used to assess and represent the pain intensity at $6,12,18$ and 24 h postsurgery (Fig. 2). The patients were instructed to select a score that was specific to the pain they were experiencing, where 0 indicates no pain, 1-3 indicates a moderate and tolerable pain, 4-6 indicates an evident pain that affects sleep but is tolerable following a simple intervention (distracting attention by reading, watching TVor listening to music) and 7-10 indicates a progressively intense pain that is intolerable and requires measures for relief.

Anesthetic dose. Both groups were compared regarding the doses of ketamine and propofol.

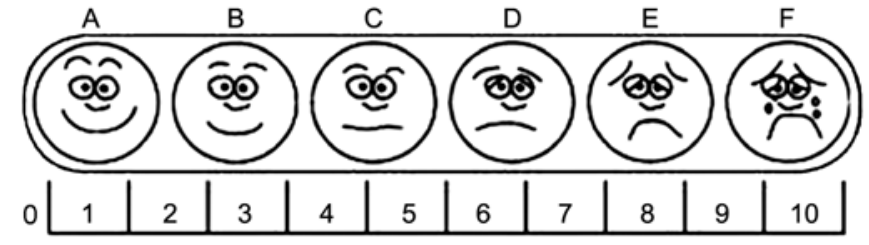

Figure 2. Visual analog scale. There are 11 numbers ranging from 0 to 10.0 , no pain; $1-3$, moderate and tolerable pain; $4-6$, evident pain affecting sleep but tolerable after simple treatment; and 7-10, progressively intensive pain that is intolerable and requires measures for its relief.

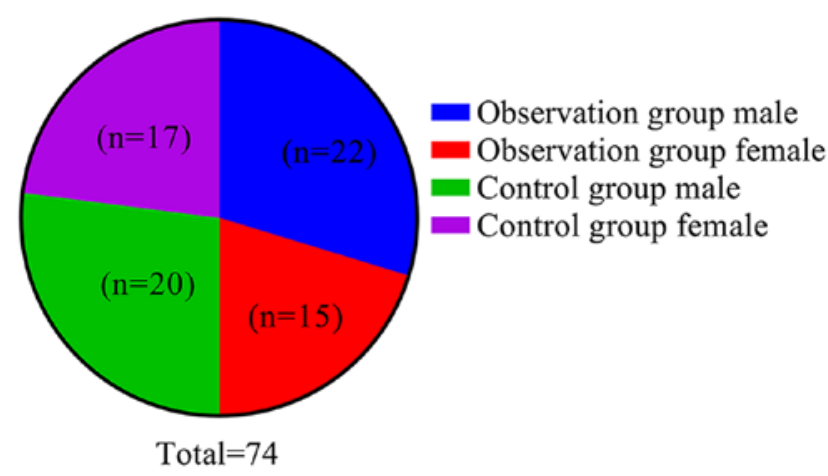

Figure 3. Number of males and females in the observation and control groups The observation group included 22 males $(59.46 \%)$ and 15 females $(40.54 \%)$, whereas the control group included 20 (54.05\%) and 17 (45.95\%), respectively, which was not significantly different between and within groups $(\mathrm{P}>0.05)$.

Adverse reactions. Both groups were compared in terms of the incidence of adverse reactions, such as restlessness, vomiting, bucking, hypotension and bradycardia, during surgery and the anesthesia recovery period.

Statistical analysis. Statistical analysis was performed using SPSS version 22.0 (IBM Corp.). Numerical data are presented as the mean \pm standard deviation, and were compared using independent samples Student's t-test for data that were normally distributed and Mann-Whitney $U$ test for non-continuous variables. Nominal data are presented as $\mathrm{n}(\%)$, and were compared between groups using the $\chi^{2}$ test or Fisher's exact test. Multipoint comparisons within and among groups were performed using mixed ANOVA and the F-test. The Tamhane test was used for post hoc test. $\mathrm{P}<0.05$ was considered to indicate a statistically significant difference.

\section{Results}

Comparison of patient characteristics. The observation and control groups exhibited no significant differences in terms of sex (Fig. 3), mean age, height, body weight and duration of surgery $(\mathrm{P}>0.05$; Table I).

Comparison of vital signs. Both the observation and control groups showed no significant difference in HR and MAP levels at time-points $\mathrm{T} 1, \mathrm{~T} 2, \mathrm{~T} 3$ and T4 ( $\mathrm{P}>0.05)$. There was no significant difference in HR and MAP levels between the observation and control groups at time-points T1, T2 and T3 ( $\mathrm{P}>0.05)$, while the HR and MAP levels in the observation group at T4 were lower than those in the control group $(\mathrm{P}<0.05$; Table II). 
Table I. General characteristics of the study subjects.

\begin{tabular}{|c|c|c|c|c|}
\hline Characteristics & Observation group $(n=37)$ & Control group $(n=37)$ & $\mathrm{t} / \chi^{2}$ value & P-value \\
\hline \multicolumn{5}{|l|}{ Sex, n (\%) } \\
\hline Male & $22(59.46)$ & $20(54.05)$ & 0.220 & 0.639 \\
\hline Female & $15(40.54)$ & $17(45.95)$ & & \\
\hline Age, years & $7.26 \pm 3.64$ & $7.84 \pm 3.91$ & 0.294 & 0.162 \\
\hline Height, cm & $112.64 \pm 10.27$ & $114.75 \pm 11.43$ & 0.835 & 0.406 \\
\hline Body weight, kg & $29.86 \pm 2.49$ & $30.44 \pm 2.61$ & 0.978 & 0.331 \\
\hline Duration of surgery, min & $40.45 \pm 12.35$ & $41.57 \pm 13.40$ & 0.374 & 0.710 \\
\hline
\end{tabular}

Data are presented as mean $\pm \mathrm{SD}$ or $\mathrm{n}(\%)$.

Table II. Vital signs at different time-points of anesthesia.

\begin{tabular}{|c|c|c|c|c|c|}
\hline Group & Time-point & HR (beats/min) & MAP (mmHg) & t-value (HR/MAP) & P-value (HR/MAP) \\
\hline \multirow[t]{4}{*}{ Observation group $(n=37)$} & $\mathrm{T} 1$ & $117.06 \pm 11.58$ & $72.89 \pm 8.25$ & $0.865 / 0.724$ & $0.271 / 0.625$ \\
\hline & $\mathrm{T} 2$ & $112.34 \pm 12.85$ & $71.03 \pm 6.34$ & & \\
\hline & $\mathrm{T} 3$ & $111.07 \pm 12.92$ & $72.16 \pm 5.24$ & & \\
\hline & $\mathrm{T} 4$ & $105.54 \pm 9.83$ & $69.31 \pm 6.25$ & & \\
\hline \multirow[t]{4}{*}{ Control group $(n=37)$} & $\mathrm{T} 1$ & $113.57 \pm 12.16$ & $73.46 \pm 5.19$ & $0.859 / 0.234$ & $0.152 / 0.321$ \\
\hline & $\mathrm{T} 2$ & $110.37 \pm 8.27$ & $70.31 \pm 5.27$ & & \\
\hline & T3 & $109.33 \pm 10.16$ & $73.29 \pm 6.15$ & & \\
\hline & $\mathrm{T} 4$ & $117.24 \pm 10.31$ & $77.08 \pm 6.52$ & & \\
\hline t-value $1_{\text {(intergroup T1) }}$ & & 0.594 & 0.421 & & \\
\hline P-value $1_{\text {(intergroup T1) }}$ & & 0.163 & 0.382 & & \\
\hline t-value 2 (intergroup T2) & & 0.958 & 0.758 & & \\
\hline P-value $2_{\text {(intergroup T2) }}$ & & 0.421 & 0.265 & & \\
\hline t-value $3_{\text {(intergroup T3) }}$ & & 0.286 & 0.362 & & \\
\hline P-value 3 (intergroup T3) & & 0.185 & 0.421 & & \\
\hline t-value $4_{\text {(intergroup T4) }}$ & & 5.829 & 6.382 & & \\
\hline P-value $4_{\text {(intergroup T4) }}$ & & $<0.001$ & $<0.001$ & & \\
\hline
\end{tabular}

Data are presented as the mean $\pm \mathrm{SD}$. $\mathrm{t} 1$ and $\mathrm{P} 1$ are the comparison between the observation group and the control group at T1 time-points; $\mathrm{t} 2$ and $\mathrm{P} 2$ are the comparison between the observation group and the control group at T2 time-points; $\mathrm{t} 3$ and $\mathrm{P} 3$ are the comparison between the observation group and the control group at T3 time-points; $\mathrm{t} 4$ and $\mathrm{P} 4$ are the comparison between the observation group and the control group at $\mathrm{T} 4$ time-points .

Comparison of sedative effects. No statistically significant difference was observed in the proportion of patients with sedation at Ramsay levels 1-6 between the observation and the control group (P>0.05; Table III and Fig. 4).

Comparison of anesthetic effects. The use of procaine combined with ketamine and propofol for epidural anesthesia resulted in a shorter time for the onset of the anesthetic effect and for the disappearance of pain, and a longer time for the recovery of pain sensation compared with that in the control group, in which ketamine and propofol were administered (all $\mathrm{P}<0.01$; Table IV).

Comparison of pain intensity. After epidural anesthesia using procaine combined with ketamine and propofol, the observation group exhibited a lower VAS score compared with the control group at $6,12,18$ and 24 h postsurgery (all $\mathrm{P}<0.01$; Table V).
Comparison of the anesthetic dose. The observation group received lower doses of ketamine and propofol compared with the control group based on the ketamine and propofol amounts that were required for anesthesia (both $\mathrm{P}<0.01$; Table VI).

Comparison of the incidence of adverse reactions. An incidence of adverse reactions of $8.11 \%$ was noted in the observation group during surgery and the anesthesia recovery period, which was lower compared with that in the control group (29.73\%; $\mathrm{P}<0.05$; Table VII).

\section{Discussion}

Children have been indicated to differ from adults in terms of physiology and psychology, and to be more sensitive to stress and pain, which results in a lower level of cooperation in surgeries that produce pain and a high possibility of restlessness (12). The 
Table III. Sedative effects of anesthesia.

\begin{tabular}{lccrrrrrrr}
\hline & \multicolumn{7}{c}{ Ramsay levels, $\mathrm{n}(\%)$} \\
\cline { 2 - 7 } Group & Level 1 & Level 2 & \multicolumn{1}{c}{ Level 3 } & Level 4 & Level 5 & Level 6 & $\chi^{2}$ value & P-value \\
\hline Observation group (n=37) & $0(0.00)$ & $2(5.41)$ & $5(13.51)$ & $10(27.03)$ & $13(35.14)$ & $7(18.92)$ & 7.638 & 0.156 \\
Control group (n=37) & $2(5.41)$ & $6(16.22)$ & $10(27.03)$ & $7(18.92)$ & $8(21.62)$ & $4(10.81)$ & & \\
\hline
\end{tabular}

Data are presented as $\mathrm{n}(\%)$.

Table IV. Time effects of anesthesia.

\begin{tabular}{lcccc}
\hline Group & $\mathrm{n}$ & $\begin{array}{c}\text { Time for anesthesia } \\
\text { to take effect, min }\end{array}$ & $\begin{array}{c}\text { Time for pain to } \\
\text { disappear, min }\end{array}$ & $\begin{array}{c}\text { Time for pain sense } \\
\text { to recover, min }\end{array}$ \\
\hline Observation group & 37 & $1.98 \pm 0.45$ & $4.22 \pm 0.69$ & $38.25 \pm 3.67$ \\
Control group & 37 & $4.32 \pm 0.73$ & $6.95 \pm 0.82$ & $23.42 \pm 2.73$ \\
t-value & 16.600 & 15.495 & 19.722 \\
P-value & $<0.001$ & $<0.001$ & $<0.001$
\end{tabular}

Data are presented as the mean $\pm \mathrm{SD}$.

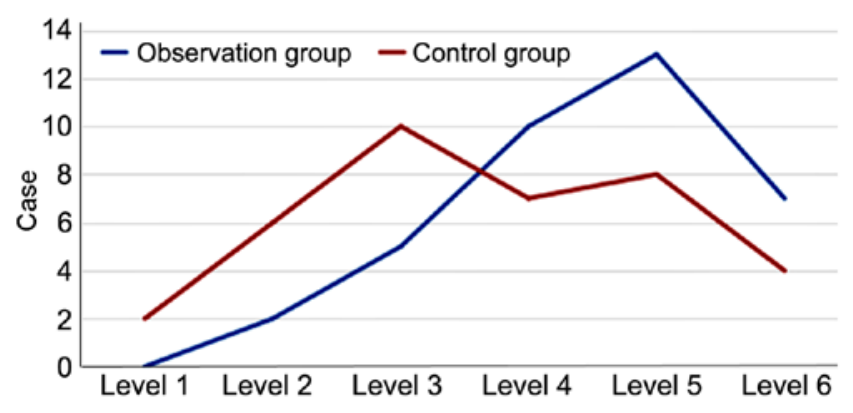

Figure 4. Levels of sedative effects in the observation and control groups. In the observation group, patients at levels $1,2,3,4,5$ and 6 accounted for 0 , $5.41,13.51,27.03,35.14$ and $18.92 \%$ of the cohort, respectively, whereas in the control group they accounted for 5.41, 16.22, 27.03, 18.92, 21.62 and $10.81 \%$ of the cohort, respectively. The observation group exhibited a lower proportion of patients at levels 1-3 and a higher proportion of patients at levels 4-6 compared with the control group, albeit with no significant difference $(\mathrm{P}>0.05)$.

differences between children and adults also include a straighter spine, a narrower epidural space and thinner neurosheaths, which have collectively been reported to contribute to the rapid release and enhanced effects of anesthetics observed in children (13). Although epidural anesthesia is increasingly considered to be suitable for delivering anesthesia to children during surgery, the varied anesthetic options available offer a challenge to the safety of anesthesia, which should be closely monitored.

Ketamine, which is a derivative of phencyclidine and a dissociative anesthetic, is the only intravenous anesthetic that has been reported to exert analgesic effects in safe doses (14). In addition to causing amnesia, ketamine has been indicated to efficiently and safely alleviate pain in patients and shorten the time required to regain consciousness; therefore, it is widely applied in pediatric intravenous anesthesia (15). However, ketamine has only been indicated to function at the body surface rather than
Table V. Visual analog scale scores at different time-points.

\begin{tabular}{lccccc}
\hline & & \multicolumn{4}{c}{ Time after surgery } \\
\cline { 3 - 6 } Group & $\mathrm{n}$ & $6 \mathrm{~h}$ & $12 \mathrm{~h}$ & $18 \mathrm{~h}$ & $24 \mathrm{~h}$ \\
\hline Observation group & 37 & $2(1-4)$ & $3(1-6)$ & $4(2-6)$ & $4(2-7)$ \\
Control group & 37 & $3(1-6)$ & $4(2-7)$ & $5(2-8)$ & $5(2-9)$ \\
t-value & & 2.986 & 3.626 & 3.754 & 3.781 \\
P-value & & 0.025 & 0.012 & 0.009 & 0.011 \\
\hline
\end{tabular}

Data are presented as median (interquartile range).

Table VI. Dosages of ketamine and propofol used for anesthesia.

\begin{tabular}{lccc}
\hline Group & $\mathrm{n}$ & Ketamine, mg & Propofol, mg \\
\hline Observation group & 37 & $71.06 \pm 6.83$ & $70.51 \pm 7.27$ \\
Control group & 37 & $81.49 \pm 7.41$ & $97.58 \pm 8.33$ \\
t-value & & 6.296 & 14.893 \\
P-value & $<0.001$ & $<0.001$ \\
\hline
\end{tabular}

Data are presented as the mean $\pm \mathrm{SD}$.

in the internal organs, and to only be adequate for short-term surgeries, with no guarantee of success being provided for long-term surgeries (16). Furthermore, Zeballos et al (17) demonstrated that ketamine alone was unable to adequately relax muscles and ease pain, and that a higher dose was required for anesthesia; however, this may result in increased oral secretion, 
Table VII. Incidence of adverse reactions during surgery and anesthesia recovery period.

Adverse reactions, $\mathrm{n}(\%)$

\begin{tabular}{lcccccc}
\cline { 2 - 6 } Group & Restlessness & Vomiting & Bucking & Hypotension & Bradycardia & Total incidence \\
\hline Observation group $(\mathrm{n}=37)$ & $1(2.70)$ & $1(2.70)$ & $1(2.70)$ & $0(0.00)$ & $0(0.00)$ & $3(8.11)$ \\
Control group $(\mathrm{n}=37)$ & $2(5.41)$ & $4(10.81)$ & $3(8.11)$ & $1(2.70)$ & $1(2.70)$ & $11(29.73)$ \\
$\chi^{2}$-value & & & & & & 5.628 \\
P-value & & & & & & 0.018
\end{tabular}

Data are presented as $\mathrm{n}(\%)$.

glossocoma, prominent agitation during the recovery period and even respiratory depression in serious cases. Consequently, ketamine is clinically combined with propofol for anesthesia to reduce the side effects and the dose of ketamine required (18). Hayes et al (19) reported that children who were administered ketamine and propofol for anesthesia had a lower likelihood of being restless during the recovery period compared with those who received ketamine alone.

The present study focused on the addition of procaine to the combination of ketamine and propofol for epidural anesthesia. Compared with the control group, who were administered ketamine and propofol, the HR and MAP levels following epidural administration (T4) were lower in the observation group $(\mathrm{P}<0.01)$, while there was no significant differences in $\mathrm{HR}$ and MAP levels between the two groups before anesthesia (T1), following anesthesia (T2) and before epidural administration (T3) $(\mathrm{P}>0.05)$, indicating that throughout the entire anesthesia process, HR and MAP in the observation group did not fluctuate significantly, while the HR and MAP of the control group increased following epidural administration. Moreover, the observation group exhibited an incidence of adverse reactions of $8.11 \%$, which was lower compared with that of the control group $(29.73 \%)(\mathrm{P}<0.01)$, suggesting that the combination of procaine with ketamine and propofol can effectively control the fluctuations in vital signs and adverse reactions during surgery, and the recovery period, thereby guaranteeing the safety of the anesthesia. This observation may be attributed to the ability of procaine to suppress cardiovascular excitation, extend the recovery period that is associated with ketamine and alleviate the inhibition of autonomic nerves by propofol, thereby maintaining a stable circulation and anesthetic effect (20). In the current study, the observation group required a shorter time for the onset of the anesthetic effect and the disappearance of pain, and a longer time for the recovery of pain sensation $(\mathrm{P}<0.01)$. The study by Giudici et al (21) indicated that following the combined application of procaine in epidural anesthesia, the time for the onset of the anesthetic effect and the disappearance of pain after anesthesia were shortened, and the recovery of pain sensation postsurgery was prolonged compared with the patients who were not administrated with procaine, which were consistent with the results of the present study. However, there are certain differences between the specific data of the two studies, which may be attributed to variation in the included subjects, the specific drug dose and the pain evaluation criteria. In contrast to the study by Giudici et al (21), the present study defined the inclusion criteria more accurately, and the patients were subjected to the same type of surgery, which may considerably reduce the influence of the type of surgery on the results.

Procaine is a local benzoate anesthetic (22). Yilbas et al (23) reported that chloroprocaine inhibited the sensory and motor nerves for a shorter period compared with lidocaine, ensuring that children regained consciousness and were able to engage in activities at the earliest. By contrast, Jalili and Saeedi (24) compared procaine with lidocaine and demonstrated that as local anesthesia, procaine reduced the incidence of transient neurological syndrome. In the present study, no statistical difference was observed in the proportion of patients with sedation at Ramsay levels 1- 6 between the observation and control groups $(\mathrm{P}>0.05)$, indicating that the two methods of anesthesia resulted in a similar sedative effect. This finding is in contrast with the study by Ying et al (25), in which the combined use of procaine for local anesthesia resulted in better sedative effects. The present study also reported lower VAS scores in the observation group at $6,12,18$ and $24 \mathrm{~h}(\mathrm{P}<0.01)$, which is consistent with the findings of Wu et al (26), indicating that procaine in combination with other local anesthetics can extend the analgesia time and reduce the pain intensity post surgery.

Finally, in the present study, the observation group received a lower dose of ketamine and propofol compared with the control group $(\mathrm{P}<0.01)$, indicating that the combined use of procaine for pediatric epidural anesthesia can exert better sedative and pain relief effects and reduce the doses of ketamine and propofol that are required. This may be attributed to the characteristics of procaine, including the limited number of toxic reactions, the efficient hydrolysis by pseudocholinesterase in serum, and the reduced time for the anesthetic effect, the achievement of analgesia and the recovery of movement $(27,28)$.

In conclusion, the current study indicated that procaine combined with ketamine and propofol in pediatric epidural anesthesia may be more commonly used considering its advantages in accelerating the anesthesia process, improving the anesthetic effects and guaranteeing the safety of the anesthesia.

However, a small number of patients were included in the present study, who were characterized by a narrow age range, thereby potentially hampering the generalizability of the results. Future long-term comprehensive analyses should prioritize the inclusion of a larger number of patients to additionally explore the advantages of the combined use of procaine with ketamine and propofol in pediatric epidural anesthesia.

\section{Acknowledgements}

Not applicable. 


\section{Funding}

No funding was received.

\section{Availability of data and materials}

The datasets used and/or analyzed during the current study are available from the corresponding author on reasonable request.

\section{Authors' contributions}

$\mathrm{HH}, \mathrm{YL}$ and ZG conceived and designed the research, and interpreted the experimental results. $\mathrm{HH}, \mathrm{YL}, \mathrm{ZG}$ and XW performed the experiments, analyzed the data, prepared the figures and drafted the manuscript. XW edited and revised the manuscript. All authors read and approved the final version of manuscript.

\section{Ethics approval and consent to participate}

The present study was approved by the Ethics Committee of Hong Hui Hospital, Xi'an Jiaotong University College of Medicine (approval no. ChiCTR1800013484). All patients and their families agreed to participate in the experiment, and parents provided written informed consent for all patients.

\section{Patient consent for publication}

Not applicable.

\section{Competing interests}

The authors declare that they have no competing interests.

\section{References}

1. Moore AD and Anghelescu DL: Erratum to: Emergence delirium in pediatric anesthesia. Paediatr Drugs 19: 267, 2017.

2. Kaniyil S, Smithamol PB, Joseph E, Krishnadas A and Ramadas KT: A survey of current practice of supraglottic airway devices in pediatric anesthesia from India. Anesth Essays Res 11: 578-582, 2017.

3. Cheng D, Liu L and Hu Z: Prevention of anesthesia-induced injection pain of propofol in pediatric anesthesia. Pak J Med Sci 33: 752-756, 2017.

4. Frawley G, Bell G, Disma N, Withington DE, de Graaff JC Morton NS, McCann ME, Arnup SJ, Bagshaw O, Wolfler A, et al Predictors of failure of awake regional anesthesia for neonatal hernia repair: Data from the general anesthesia compared to spinal anesthesia study-comparing apnea and neurodevelopmental outcomes. Anesthesiology 123: 55-65, 2015.

5. Leemans B, Stout TA, Soom AV and Gadella BM: pH-dependent effects of procaine on equine gamete activation. Biol Reprod 101: 1056-1074, 2019.

6. Förster JG and Rosenberg PH: Revival of old local anesthetics for spinal anesthesia in ambulatory surgery. Curr Opin Anaesthesiol 24: 633-637, 2011.

7. Wildsmith JA: Reactions to procaine after caudal injection. Reg Anesth Pain Med 43: 446, 2018.

8. Mak PH, Campbell RC and Irwin MG; American Society of Anesthesiologists: The ASA physical status classification: Inter-observer consistency. American society of anesthesiologists. Anaesth Intensive Care 30: 633-640, 2002.

9. Korzh EA, Klymenko NA and Smolin SK: Bioregeneration of the activated carbon layer spent in the dynamics of procaine biofiltration. J Water Chem Technol 39: 103-107, 2017.

10. Mondello E, Siliotti R, Noto G, Cuzzocrea E, Scollo G, Trimarchi G and Venuti FS: Bispectral Index in ICU: Correlation with ramsay score on assessment of sedation level. J Clin Monit Comput 17: 271-277, 2002.
11. Knop C, Oeser M, Bastian L, Lange U, Zdichavsky M and Blauth M: Development and validation of the visual analogue scale (VAS) spine score. Unfallchirurg 104: 488-497, 2001 (In German).

12. Guo J, Jin X, Wang H, Yu J, Zhou X, Cheng Y, Tao Q, Liu L and Zhang J: Emergence and recovery characteristics of five common anesthetics in pediatric anesthesia: A network meta-analysis. Mol Neurobiol 54: 4353-4364, 2017.

13. Walker BJ, Long JB, Sathyamoorthy M, Birstler J, Wolf C, Bosenberg AT, Flack SH, Krane EJ, Sethna NF, Suresh S, et al: Complications in pediatric regional anesthesia: An analysis of more than 100,000 blocks from the pediatric regional anesthesia network. Anesthesiology 129: 721-732, 2018.

14. Lefevre A and Schnepper G: Development of Harlequin Syndrome following placement of thoracic epidural anesthesia in a pediatric patient undergoing Nuss procedure. Clin Case Rep 5: 1523-1525, 2017.

15. Hambrecht-Wiedbusch VS, Li D and Mashour GA: Paradoxical emergence: Administration of subanesthetic ketamine during isoflurane anesthesia induces burst suppression but accelerates recovery. Anesthesiology 126: 482, 2017.

16. Tosh P, Rajan S, Puthenveettil N and Kumar L: Oral clonidine premedication attenuates hemodynamic responses of ketamine during total intravenous anesthesia. Anesth Essays Res 11: 617-620, 2017.

17. Zeballos JL, Lirk P and Rathmell JP: Low-dose ketamine for acute pain management: A timely nudge toward multimodal analgesia. Reg Anesth Pain Med 43: 453-455, 2018.

18. Baradari AG, Alipour A, Habibi MR, Rashidaei S and Emami Zeydi A: A randomized clinical trial comparing hemodynamic responses to ketamine-propofol combination (ketofol) versus etomidate during anesthesia induction in patients with left ventricular dysfunction undergoing coronary artery bypass graft surgery. Arch Med Sci 13: 1102-1110, 2017.

19. Hayes J, Matava C, Pehora C, El-Beheiry H, Jarvis S and Finkelstein Y: Determination of the median effective dose of propofol in combination with different doses of ketamine during gastro-duodenoscopy in children: A randomised controlled trial. Br J Anaesth 121: 453-461, 2018.

20. Teunkens A, Vermeulen K, Van Gerven E, Fieuws S, Van de Velde $\mathrm{M}$ and Rex S: Comparison of 2-chloroprocaine, bupivacaine, and lidocaine for spinal anesthesia in patients undergoing knee arthroscopy in an outpatient setting: A double-blind randomized controlled trial. Reg Anesth Pain Med 41: 576-583, 2016.

21. Giudici V, Baeza S, Douet JY and Regnier A: Corneal anesthesia following application of $0.4 \%$ oxybuprocaine hydrochloride ophthalmic solution to normal feline eyes. Vet Ophthalmol 18: 141-146, 2015.

22. Bodart JF, Flament S, Browaeys E, Bertout M, Rousseau A, Gannon J and Vilain JP: MPF and procaine-induced maturation of xenopus oocyte. Biol Cell 88: 71, 1996.

23. Yilbas AA, Akca B, Buyukakkus B, Bahador Zirh E, Zeybek D, Uzumcugil F and Saricaoglu F: Procaine and saline have similar effects on articular cartilage and synovium in rat knee. BMC Anesthesiol 18: 51, 2018.

24. Jalili S and Saeedi M: Study of procaine and tetracaine in the lipid bilayer using molecular dynamics simulation. Eur Biophys J 46: 265-282, 2017.

25. Ying B, Huang H, Li H, Song M, Wu S and Ying H: Procaine inhibits proliferation and migration and promotes cell apoptosis in osteosarcoma cells by upregulation of MicroRNA-133b. Oncol Res 25: 1463-1470, 2017.

26. Wu F, Wang J, Sun J, Shen L, Liu M and Zhao E: Procaine stimulates aquaporin-5 expression in human salivary gland ductal cells via the suppression of DNA methyltransferase-1. Mol Med Rep 17: 7996-8002, 2018.

27. Hao K, Suryoprabowo S, Song S, Liu L, Zheng Q and Kuang H: Development of an immunochromatographic test strip for the detection of procaine in milk. Food Agric Immunol 29: 1150-1161, 2018.

28. Lin H, Wang Z, Shen J, Xu J and Li H: Intravenous anesthetic ketamine attenuates complete Freund's adjuvant-induced arthritis in rats via modulation of MAPKs/NF-кB. Inflamm Res 68: 147-155, 2019.
This work is licensed under a Creative Commons Attribution-NonCommercial-NoDerivatives 4.0 International (CC BY-NC-ND 4.0) License. 